\title{
Multiple Positive Solutions for Nonlinear First-Order Impulsive Dynamic Equations on Time Scales with Parameter
}

\section{Da-Bin Wang and Wen Guan}

Department of Applied Mathematics, Lanzhou University of Technology, Lanzhou, Gansu, 730050, China

Correspondence should be addressed to Da-Bin Wang, wangdb@lut.cn

Received 13 February 2009; Accepted 14 May 2009

Recommended by Victoria Otero-Espinar

By using the Leggett-Williams fixed point theorem, the existence of three positive solutions to a class of nonlinear first-order periodic boundary value problems of impulsive dynamic equations on time scales with parameter are obtained. An example is given to illustrate the main results in this paper.

Copyright (C) 2009 D.-B. Wang and W. Guan. This is an open access article distributed under the Creative Commons Attribution License, which permits unrestricted use, distribution, and reproduction in any medium, provided the original work is properly cited.

\section{Introduction}

Let $\mathbf{T}$ be a time scale, that is, $T$ is a nonempty closed subset of $R$. Let $T>0$ be fixed and $0, T$ be points in $\mathbf{T}$, an interval $(0, T)_{\mathrm{T}}$ denoting time scales interval, that is, $(0, T)_{\mathrm{T}}:=(0, T) \cap \mathbf{T}$. Other types of intervals are defined similarly. Some definitions concerning time scales can be found in [1-5].

In this paper, we are concerned with the existence of positive solutions for the following nonlinear first-order periodic boundary value problem on time scales:

$$
\begin{gathered}
x^{\Delta}(t)+p(t) x(\sigma(t))=\lambda f(t, x(\sigma(t))), \quad t \in J:=[0, T]_{\mathrm{T}}, t \neq t_{k}, k=1,2, \ldots, m, \\
x\left(t_{k}^{+}\right)-x\left(t_{k}^{-}\right)=I_{k}\left(x\left(t_{k}^{-}\right)\right), \quad k=1,2, \ldots, m, \\
x(0)=x(\sigma(T)),
\end{gathered}
$$

where $\lambda>0$ is a positive parameter, $f \in C(J \times[0, \infty),[0, \infty)), I_{k} \in C([0, \infty),[0, \infty)), p$ : $[0, T]_{\mathrm{T}} \rightarrow(0, \infty)$ is right-dense continuous, $t_{k} \in(0, T)_{\mathrm{T}}, 0<t_{1}<\cdots<t_{m}<T$, and for each 
$k=1,2, \ldots, m, x\left(t_{k}^{+}\right)=\lim _{h \rightarrow 0^{+}} x\left(t_{k}+h\right)$ and $x\left(t_{k}^{-}\right)=\lim _{h \rightarrow 0^{-}} x\left(t_{k}+h\right)$ represent the right and left limits of $x(t)$ at $t=t_{k}$.

The theory of impulsive differential equations is emerging as an important area of investigation, since it is a lot richer than the corresponding theory of differential equations without impulse effects. Moreover, such equations may exhibit several real world phenomena in physics, biology, engineering, and so forth, (see [6-8]). At the same time, the boundary value problems for impulsive differential equations and impulsive difference equations have received much attention [9-19]. On the other hand, recently, the theory of dynamic equations on time scales has become a new important branch (see, e.g., [1-5]). Naturally, some authors have focused their attention on the boundary value problems of impulsive dynamic equations on time scales [20-27]. In particular, for the first-order impulsive dynamic equations on time scales

$$
\begin{gathered}
y^{\Delta}(t)+p(t) y(\sigma(t))=f(t, y(t)), \quad t \in J:=[a, b], t \neq t_{k}, k=1,2, \ldots, m, \\
y\left(t_{k}^{+}\right)=I_{k}\left(y\left(t_{k}^{-}\right)\right), \quad k=1,2, \ldots, m, \\
y(a)=\eta
\end{gathered}
$$

where $\mathbf{T}$ is a time scale which has at least finitely-many right-dense points, $[a, b] \subset \mathbf{T}, p$ is regressive and right-dense continuous, $f: \mathrm{T} \times R \rightarrow R$ is given function, $I_{k} \in C(R, R)$. The paper [21] obtained the existence of one solution to problem (1.2) by using the nonlinear alternative of Leray-Schauder type.

In [22], Benchohra et al. considered the following impulsive boundary value problem on time scales

$$
\begin{gathered}
-y^{\Delta \Delta}(t)=f(t, y(t)), \quad t \in J:=[0,1]_{\mathrm{T}}, t \neq t_{k}, \\
y\left(t_{k}^{+}\right)-y\left(t_{k}^{-}\right)=I_{k}\left(y\left(t_{k}^{-}\right)\right) \\
y^{\Delta}\left(t_{k}^{+}\right)-y^{\Delta}\left(t_{k}^{-}\right)=\bar{I}_{k}\left(y\left(t_{k}^{-}\right)\right) \\
y(0)=y(1)=0 .
\end{gathered}
$$

They proved the existence of one solution to the problem (1.3) by applying Schaefer's fixed point theorem and the nonlinear alternative of Leray-Schauder type.

In [26], Li and Shen studied the problem (1.3). Some existence results to problem (1.3) are established by using a fixed point theorem, which is due to Krasnoselskii and Zabreiko, and the Leggett-Williams fixed point theorem.

In [27], the first author studied the problem (1.1) when $\lambda=1$. The existence of positive solutions to the problem (1.1) was obtained by means of the well-known Guo-Krasnoselskii fixed point theorem.

Recently, Sun and Li [28] considered the following periodic boundary value problem:

$$
\begin{gathered}
x^{\Delta}(t)+p(t) x(\sigma(t))=\lambda f(x(t)), \quad t \in[0, T]_{\mathrm{T}}, \\
x(0)=x(\sigma(T)) .
\end{gathered}
$$


By using the fixed point index, some existence, multiplicity and nonexistence criteria of positive solutions to the problem (1.4) were obtained for suitable $\lambda>0$.

Motivated by the results mentioned above, in this paper, we shall show that the problem (1.1) has at least three positive solutions for suitable $\lambda>0$ by using the LeggettWilliams fixed point theorem [29]. We note that for the case $\lambda=1$ and $I_{k}(x) \equiv 0, k=$ $1,2, \ldots, m$, problem (1.1) reduces to the problem studied by [30].

In the remainder of this section, we state the following theorem, which are crucial to our proof.

Let $E$ be a real Banach space and $K \subset E$ be a cone. A function $\alpha: K \rightarrow[0, \infty)$ is called a nonnegative continuous concave functional if $\alpha$ is continuous and

$$
\alpha(t x+(1-t) y) \geq t \alpha(x)+(1-t) \alpha(y)
$$

for all $x, y \in K$ and $t \in[0,1]$.

Let $a, b>0$ be constants, $K_{a}=\{x \in K:\|x\|<a\}, K(\alpha, a, b)=\{x \in K: a \leq \alpha(x),\|x\| \leq$ $b\}$.

Theorem 1.1 (see [29]). Let $A: \bar{K}_{c} \rightarrow \bar{K}_{c}$ be a completely continuous map and $\alpha$ be a nonnegative continuous concave functional on $K$ such that $\alpha(x) \leq\|x\|, \forall x \in \bar{K}_{c}$. Suppose there exist $a, b, d$ with $0<d<a<b \leq c$ such that

(i) $\{x \in K(\alpha, a, b): \alpha(x)>a\} \neq \phi$ and $\alpha(A x)>a \forall x \in K(\alpha, a, b)$;

(ii) $\|A x\|<d \forall x \in K_{d}$;

(iii) $\alpha(A x)>a, \forall x \in K(\alpha, a, c)$ with $\|A x\|>b$.

Then $A$ has at least three fixed points $x_{1}, x_{2}, x_{3}$ in $\bar{K}_{c}$ satisfying

$$
\left\|x_{1}\right\|<d, \quad a<\alpha\left(x_{2}\right), \quad\left\|x_{3}\right\|>d \quad \text { with } \alpha\left(x_{3}\right)<a .
$$

\section{Preliminaries}

Throughout the rest of this paper, we always assume that the points of impulse $t_{k}$ are rightdense for each $k=1,2, \ldots, m$.

We define

$$
\begin{gathered}
P C=\left\{x \in[0, \sigma(T)]_{\mathrm{T}} \longrightarrow R: x_{k} \in C\left(J_{k}, R\right), k=1,2, \ldots, m\right. \text { and there exist } \\
\left.x\left(t_{k}^{+}\right) \text {and } x\left(t_{k}^{-}\right) \text {with } x\left(t_{k}^{-}\right)=x\left(t_{k}\right), k=1,2, \ldots, m\right\},
\end{gathered}
$$

where $x_{k}$ is the restriction of $x$ to $J_{k}=\left(t_{k}, t_{k+1}\right]_{\mathrm{T}} \subset(0, \sigma(T)]_{\mathrm{T}}, k=1,2, \ldots, m$ and $J_{0}=$ $\left[0, t_{1}\right]_{T}, J_{m+1}=\sigma(T)$.

Let

$$
X=\{x(t): x(t) \in P C, x(0)=x(\sigma(T))\}
$$

with the norm $\|x\|=\sup _{t \in[0, \sigma(T)]_{\mathrm{T}}}|x(t)|$. Then $\mathrm{X}$ is a Banach space. 
Definition 2.1. A function $x \in P C \cap C^{1}\left(J \backslash\left\{t_{1}, t_{2}, \ldots, t_{m}\right\}, R\right)$ is said to be a solution of the problem (1.1) if and only if $x$ satisfies the dynamic equation

$$
x^{\Delta}(t)+p(t) x(\sigma(t))=\lambda f(t, x(\sigma(t))) \text { every where on } J \backslash\left\{t_{1}, t_{2}, \ldots, t_{m}\right\},
$$

the impulsive conditions

$$
x\left(t_{k}^{+}\right)-x\left(t_{k}^{-}\right)=I_{k}\left(x\left(t_{k}^{-}\right)\right), \quad k=1,2, \ldots, m,
$$

and the periodic boundary condition $x(0)=x(\sigma(T))$.

Lemma 2.2. Suppose $h:[0, T]_{\mathrm{T}} \rightarrow R$ is $r d$-continuous, then $x$ is a solution of

$$
x(t)=\lambda \int_{0}^{\sigma(T)} G(t, s) h(s) \Delta s+\sum_{k=1}^{m} G\left(t, t_{k}\right) I_{k}\left(x\left(t_{k}\right)\right), \quad t \in[0, \sigma(T)]_{\mathrm{T}},
$$

where

$$
G(t, s)= \begin{cases}\frac{e_{p}(s, t) e_{p}(\sigma(T), 0)}{e_{p}(\sigma(T), 0)-1}, & 0 \leq s \leq t \leq \sigma(T), \\ \frac{e_{p}(s, t)}{e_{p}(\sigma(T), 0)-1}, & 0 \leq t<s \leq \sigma(T),\end{cases}
$$

if and only if $x$ is a solution of the boundary value problem

$$
\begin{gathered}
x^{\Delta}(t)+p(t) x(\sigma(t))=\lambda h(t), \quad t \in J:=[0, T]_{\mathrm{T}}, t \neq t_{k}, k=1,2, \ldots, m, \\
x\left(t_{k}^{+}\right)-x\left(t_{k}^{-}\right)=I_{k}\left(x\left(t_{k}^{-}\right)\right), \quad k=1,2, \ldots, m, \\
x(0)=x(\sigma(T)) .
\end{gathered}
$$

Proof. Since the method is similar to that of in [27, Lemma 3.1], we omit it here.

Lemma 2.3. Let $G(t, s)$ be defined as Lemma 2.2, then

$$
\frac{1}{e_{p}(\sigma(T), 0)-1} \leq G(t, s) \leq \frac{e_{p}(\sigma(T), 0)}{e_{p}(\sigma(T), 0)-1} \quad \forall t, s \in[0, \sigma(T)]_{\mathrm{T}}
$$

Proof. It is obvious, so we omit it here.

Let

$$
K=\{x(t) \in X: x(t) \geq \delta\|x\|\},
$$

where $\delta=1 / e_{p}(\sigma(T), 0) \in(0,1)$. It is not difficult to verify that $K$ is a cone in $X$. 
We define an operator $\Phi: K \rightarrow X$ by

$(\Phi x)(t)=\lambda \int_{0}^{\sigma(T)} G(t, s) f(s, x(\sigma(s))) \Delta s+\sum_{k=1}^{m} G\left(t, t_{k}\right) I_{k}\left(x\left(t_{k}\right)\right), \quad t \in[0, \sigma(T)]_{\mathrm{T}}$.

By [27, Lemmas 3.3 and 3.4], it is easy to see that $\Phi: K \rightarrow K$ is completely continuous.

\section{Main Result}

Notation 1. Let

$$
\begin{array}{rlrl}
f^{0} & =\limsup _{x \rightarrow 0} \max _{t \in[0, T]_{\mathrm{T}}} \frac{f(t, x)}{x}, & I^{0}=\lim _{x \rightarrow 0} \sup \sum_{k=1}^{m} \frac{I_{k}(x)}{x}, \\
f^{\infty}=\lim _{x \rightarrow \infty} \sup \max _{t \in[0, T]_{\mathrm{T}}} \frac{f(t, x)}{x}, & I^{\infty}=\lim _{x \rightarrow \infty} \sup \sum_{k=1}^{m} \frac{I_{k}(x)}{x},
\end{array}
$$

and for $\mu>0$, we define $I_{(\mu)}=\min _{\delta \mu \leq x \leq \mu} \sum_{k=1}^{m} I_{k}(x)$.

Theorem 3.1. Assume that there exists a number $b>0$ such that the following conditions:

$\left(\mathrm{H}_{1}\right) f(t, x)>e_{p}(\sigma(T), 0) x-e_{p}(\sigma(T), 0) /\left(e_{p}(\sigma(T), 0)-1\right) I_{(b)} \geq 0$ for $\delta b \leq x \leq b, t \in[0, T]_{T} ;$

$\left(\mathrm{H}_{2}\right) f^{0}+I^{0}<\left(e_{p}(\sigma(T), 0)-1\right) / e_{p}(\sigma(T), 0), f^{\infty}+I^{\infty}<\left(e_{p}(\sigma(T), 0)-1\right) / e_{p}(\sigma(T), 0)$ hold. Then the problem (1.1) has at least three positive solutions for

$$
\frac{e_{p}(\sigma(T), 0)-1}{\sigma(T) e_{p}(\sigma(T), 0)}<\lambda<\frac{1}{\sigma(T)}
$$

Proof. Let $\alpha(x)=\min _{t \in[0, \sigma(T)]_{\mathrm{T}}} x(t)$, it is easy to see that $\alpha(x)$ is a nonnegative continuous concave functional on $K$ such that $\alpha(x) \leq\|x\|, \forall x \in \bar{K}_{c}$.

First, we assert that there exists $c>b$ such that $\Phi: \bar{K}_{c} \rightarrow \bar{K}_{c}$ is completely continuous. In fact, by the condition $f^{\infty}+I^{\infty}<\left(e_{p}(\sigma(T), 0)-1\right) / e_{p}(\sigma(T), 0)$ of $\left(\mathrm{H}_{2}\right)$, there exist $C_{0}>b$, and $0<\varepsilon<\left(\left(e_{p}(\sigma(T), 0)-1\right) / e_{p}(\sigma(T), 0)-\left(f^{\infty}+I^{\infty}\right)\right) / 2$ such that

$$
f(t, x) \leq\left(\varepsilon+f^{\infty}\right) x, \sum_{k=1}^{m} I_{k}(x) \leq\left(\varepsilon+I^{\infty}\right) x, \quad \text { for } x>C_{0} .
$$


Let $C_{1}=C_{0} / \delta$, if $x \in K,\|x\|>C_{1}$, then $x>C_{0}$ and we have

$$
\begin{aligned}
(\Phi x)(t) & =\lambda \int_{0}^{\sigma(T)} G(t, s) f(s, x(\sigma(s))) \Delta s+\sum_{k=1}^{m} G\left(t, t_{k}\right) I_{k}\left(x\left(t_{k}\right)\right) \\
& \leq \lambda \frac{e_{p}(\sigma(T), 0)}{e_{p}(\sigma(T), 0)-1} \int_{0}^{\sigma(T)}\left(\varepsilon+f^{\infty}\right)\|x\| \Delta s+\frac{e_{p}(\sigma(T), 0)}{e_{p}(\sigma(T), 0)-1}\left(\varepsilon+I^{\infty}\right)\|x\| \\
& =\left[\lambda \frac{e_{p}(\sigma(T), 0)}{e_{p}(\sigma(T), 0)-1} \sigma(T)\left(\varepsilon+f^{\infty}\right)+\frac{e_{p}(\sigma(T), 0)}{e_{p}(\sigma(T), 0)-1}\left(\varepsilon+I^{\infty}\right)\right]\|x\| \\
& <\|x\| .
\end{aligned}
$$

Take $\bar{K}_{C_{1}}=\left\{x \mid x \in K,\|x\| \leq C_{1}\right\}$, then the set $\bar{K}_{C_{1}}$ is a bounded set. According to that $\Phi$ is completely continuous, then $\Phi$ maps bounded sets into bounded sets and there exists a number $C_{2}$ such that

$$
\|\Phi x\| \leq C_{2} \text { for any } x \in \bar{K}_{C_{1}} \text {. }
$$

If $C_{2} \leq C_{1}$, we deduce that $\Phi: \bar{K}_{C_{1}} \rightarrow \bar{K}_{C_{1}}$ is completely continuous. If $C_{1}<C_{2}$, then from (3.4), we know that for any $x \in \bar{K}_{C_{2}} \backslash \bar{K}_{C_{1}},\|x\|>C_{1}$ and $\|\Phi x\|<\|x\| \leq C_{2}$ hold. Then we have $\Phi: \bar{K}_{C_{2}} \rightarrow \bar{K}_{C_{2}}$ is completely continuous. Take $c=\max \left\{C_{1}, C_{2}\right\}$, then $c>b$ and $\Phi: \bar{K}_{c} \rightarrow \bar{K}_{c}$ are completely continuous.

Second, we assert that $\{x \in K(\alpha, \delta b, b): \alpha(x)>\delta b\} \neq \phi$ and $\alpha(A x)>\delta b$ for all $x \in$ $K(\alpha, \delta b, b)$.

In fact, take $x \equiv(b+\delta b) / 2$, so $x \in\{x \in K(\alpha, \delta b, b): \alpha(x)>\delta b\}$. Moreover, for $x \in K(\alpha, \delta b, b)$, then $\alpha(x) \geq \delta b$ and we have

$$
\begin{aligned}
\alpha(\Phi x)= & \min _{t \in[0, \sigma(T)]_{\mathrm{T}}}\left[\lambda \int_{0}^{\sigma(T)} G(t, s) f(s, x(\sigma(s))) \Delta s+\sum_{k=1}^{m} G\left(t, t_{k}\right) I_{k}\left(x\left(t_{k}\right)\right)\right] \\
\geq & \frac{\lambda}{e_{p}(\sigma(T), 0)-1} \cdot \sigma(T)\left(e_{p}(\sigma(T), 0) \alpha(x)-\frac{e_{p}(\sigma(T), 0)}{e_{p}(\sigma(T), 0)-1} I_{(b)}\right) \\
& +\frac{1}{e_{p}(\sigma(T), 0)-1} I_{(b)} \\
> & \alpha(x) \geq \delta b .
\end{aligned}
$$

Third, we assert that there exist $0<d<\delta b$ such that $\|\Phi x\|<d$ if $x \in K_{d}$.

Indeed, by the condition $f^{0}+I^{0}<\left(e_{p}(\sigma(T), 0)-1\right) / e_{p}(\sigma(T), 0)$ of $\left(\mathrm{H}_{2}\right)$, there exist $0<d<\delta b$, and $0<\varepsilon<\left(\left(e_{p}(\sigma(T), 0)-1\right) / e_{p}(\sigma(T), 0)-\left(f^{0}+I^{0}\right)\right) / 2$ such that

$$
f(t, x) \leq\left(\varepsilon+f^{0}\right) x, \sum_{k=1}^{m} I_{k}(x) \leq\left(\varepsilon+I^{0}\right) x, \quad \text { for } 0 \leq x \leq d .
$$


Then $x \in K_{d}$, we get

$$
\begin{aligned}
(\Phi x)(t) & =\lambda \int_{0}^{\sigma(T)} G(t, s) f(s, x(\sigma(s))) \Delta s+\sum_{k=1}^{m} G\left(t, t_{k}\right) I_{k}\left(x\left(t_{k}\right)\right) \\
& \leq \lambda \frac{e_{p}(\sigma(T), 0)}{e_{p}(\sigma(T), 0)-1} \int_{0}^{\sigma(T)}\left(\varepsilon+f^{0}\right) x(s) \Delta s+\frac{e_{p}(\sigma(T), 0)}{e_{p}(\sigma(T), 0)-1}\left(\varepsilon+I^{0}\right)\|x\| \\
& \leq\left[\lambda \frac{e_{p}(\sigma(T), 0)}{e_{p}(\sigma(T), 0)-1}\left(\varepsilon+f^{0}\right) \sigma(T)+\frac{e_{p}(\sigma(T), 0)}{e_{p}(\sigma(T), 0)-1}\left(\varepsilon+I^{0}\right)\right]\|x\| \\
& <\frac{e_{p}(\sigma(T), 0)}{e_{p}(\sigma(T), 0)-1}\left(f^{0}+I^{0}+2 \varepsilon\right)\|x\| \\
& <\|x\|<d .
\end{aligned}
$$

Finally, we assert that $\alpha(\Phi x)>\delta b$ if $x \in K(\alpha, \delta b, c)$ and $\|\Phi x\|>b$.

To do this, if $x \in K(\alpha, \delta b, c)$ and $\|\Phi x\|>b$, then

$$
\alpha(\Phi x) \geq(\Phi x)(t) \geq \delta\|\Phi x\|>\delta b
$$

To sum up, all the hypotheses of Theorem 1.1 are satisfied by taking $a=\delta b$. Hence $\Phi$ has at least three fixed points, that is, the problem (1.1) has at least three positive solutions $x_{1}, x_{2}$ and $x_{3}$ such that

$$
\left\|x_{1}\right\|<d, a<\alpha\left(x_{2}\right), \quad\left\|x_{3}\right\|>d \text { with } \alpha\left(x_{3}\right)<a .
$$

Corollary 3.2. Using $\left(H_{3}\right) f^{0}=I^{0}=f^{\infty}=I^{\infty}=0$, instead of $\left(H_{2}\right)$ in Theorem 3.1, the conclusion of Theorem 3.1 remains true.

\section{Example}

Example 4.1. Let $\mathbf{T}=[0,1] \cup[2,3]$. We consider the following problem on $\mathbf{T}$ :

$$
\begin{gathered}
x^{\Delta}(t)+x(\sigma(t))=\lambda f(t, x(\sigma(t))), \quad t \in[0,3]_{\mathrm{T}}, t \neq \frac{1}{2} \\
x\left(\frac{1}{2}^{+}\right)-x\left(\frac{1^{-}}{2}\right)=I\left(x\left(\frac{1}{2}\right)\right) \\
x(0)=x(3)
\end{gathered}
$$


where $\mathcal{\lambda}>0$ is a positive parameter, $p(t) \equiv 1, T=3, m=1$, and

$$
\begin{aligned}
f(t, x) & = \begin{cases}9 e^{6}(t+1) x^{2}, & {[0,1],} \\
9 e^{6}(t+1) x^{1 / 2}, & {[1, \infty),}\end{cases} \\
I(x) & = \begin{cases}x^{2}, & {[0,1],} \\
x^{1 / 2}, & {[1, \infty) .}\end{cases}
\end{aligned}
$$

Taking $b=1$, then by $\delta=1 /\left(2 e^{2}\right)$ it is easy to see that $I_{(b)}=\min _{\delta b \leq x \leq b} I(x)=1 /\left(4 e^{4}\right)$. So, $\forall x \in[\delta b, b]=\left[1 /\left(2 e^{2}\right), 1\right]$, we have $f(t, x) \geq\left(9 / 4 e^{2}\right)>2 e^{2}-1 /\left[\left(2 e^{2}-1\right) 2 e^{2}\right] \geq 2 e^{2} x-$ $\left(2 e^{2}\right) /\left(2 e^{2}-1\right) 1 /\left(4 e^{4}\right)=e_{p}(\sigma(T), 0) x-e_{p}(\sigma(T), 0) /\left(e_{p}(\sigma(T), 0)-1\right) I_{(b)}$. Obviously, we have $f^{0}=I^{0}=f^{\infty}=I^{\infty}=0$.

Therefore, together with Corollary 3.2, it follows that the problem (4.1) has at least three positive solutions for $\left(2 e^{2}-1\right) /\left(6 e^{2}\right)<\lambda<1 / 3$.

\section{Acknowledgment}

The authors express their gratitude to the anonymous referee for his/her valuable suggestions.

\section{References}

[1] R. P. Agarwal and M. Bohner, "Basic calculus on time scales and some of its applications," Results in Mathematics, vol. 35, no. 1-2, pp. 3-22, 1999.

[2] M. Bohner and A. Peterson, Dynamic Equations on Time Scales: An Introduction with Applications, Birkhäuser, Boston, Mass, USA, 2001.

[3] M. Bohner and A. Peterson, Eds., Advances in Dynamic Equations on Time Scales, Birkhäuser, Boston, Mass, USA, 2003.

[4] S. Hilger, "Analysis on measure chains-a unified approach to continuous and discrete calculus," Results in Mathematics, vol. 18, no. 1-2, pp. 18-56, 1990.

[5] V. Lakshmikantham, S. Sivasundaram, and B. Kaymakcalan, Dynamic Systems on Measure Chains, vol. 370 of Mathematics and Its Applications, Kluwer Academic Publishers, Dordrecht, The Netherlands, 1996.

[6] D. D. Baĭnov and P. S. Simeonov, Systems with Impulse Effect: Stability, Theory and Applications, Ellis Horwood Series: Mathematics and Its Applications, Ellis Horwood, Chichester, UK, 1989.

[7] D. D. Bainov and P. S. Simeonov, Impulsive Differential Equations: Periodic Solutions and Applications, Longman Scientific and Technical, Harlow, UK, 1993.

[8] V. Lakshmikantham, D. D. BaĬnov, and P. S. Simeonov, Theory of Impulsive Differential Equations, vol. 6 of Series in Modern Applied Mathematics, World Scientific, Teaneck, NJ, USA, 1989.

[9] R. P. Agarwal and D. O'Regan, "Multiple nonnegative solutions for second order impulsive differential equations," Applied Mathematics and Computation, vol. 114, no. 1, pp. 51-59, 2000.

[10] Z. He and J. Yu, "Periodic boundary value problem for first-order impulsive functional differential equations," Journal of Computational and Applied Mathematics, vol. 138, no. 2, pp. 205-217, 2002.

[11] Z. He and X. Zhang, "Monotone iterative technique for first order impulsive difference equations with periodic boundary conditions," Applied Mathematics and Computation, vol. 156, no. 3, pp. 605620, 2004.

[12] J.-L. Li and J.-H. Shen, "Existence of positive periodic solutions to a class of functional differential equations with impulses," Mathematica Applicata, vol. 17, no. 3, pp. 456-463, 2004.

[13] J. Li, J. J. Nieto, and J. Shen, "Impulsive periodic boundary value problems of first-order differential equations," Journal of Mathematical Analysis and Applications, vol. 325, no. 1, pp. 226-236, 2007. 
[14] J. Li and J. Shen, "Positive solutions for first order difference equations with impulses," International Journal of Difference Equations, vol. 1, no. 2, pp. 225-239, 2006.

[15] Y. Li, X. Fan, and L. Zhao, "Positive periodic solutions of functional differential equations with impulses and a parameter," Computers \& Mathematics with Applications, vol. 56, no. 10, pp. 2556-2560, 2008.

[16] J. J. Nieto, "Basic theory for nonresonance impulsive periodic problems of first order," Journal of Mathematical Analysis and Applications, vol. 205, no. 2, pp. 423-433, 1997.

[17] J. J. Nieto, "Impulsive resonance periodic problems of first order," Applied Mathematics Letters, vol. 15, no. 4, pp. 489-493, 2002.

[18] J. J. Nieto, "Periodic boundary value problems for first-order impulsive ordinary differential equations," Nonlinear Analysis: Theory, Methods \& Applications, vol. 51, no. 7, pp. 1223-1232, 2002.

[19] A. S. Vatsala and Y. Sun, "Periodic boundary value problems of impulsive differential equations," Applicable Analysis, vol. 44, no. 3-4, pp. 145-158, 1992.

[20] A. Belarbi, M. Benchohra, and A. Ouahab, "Existence results for impulsive dynamic inclusions on time scales," Electronic Journal of Qualitative Theory of Differential Equations, vol. 2005, no. 12, pp. 1-22, 2005.

[21] M. Benchohra, J. Henderson, S. K. Ntouyas, and A. Ouahab, “On first order impulsive dynamic equations on time scales," Journal of Difference Equations and Applications, vol. 10, no. 6, pp. 541-548, 2004.

[22] M. Benchohra, S. K. Ntouyas, and A. Ouahab, "Existence results for second order boundary value problem of impulsive dynamic equations on time scales," Journal of Mathematical Analysis and Applications, vol. 296, no. 1, pp. 65-73, 2004.

[23] F. Geng, Y. Xu, and D. Zhu, "Periodic boundary value problems for first-order impulsive dynamic equations on time scales," Nonlinear Analysis: Theory, Methods E Applications, vol. 69, no. 11, pp. 40744087, 2008.

[24] J. R. Graef and A. Ouahab, "Extremal solutions for nonresonance impulsive functional dynamic equations on time scales," Applied Mathematics and Computation, vol. 196, no. 1, pp. 333-339, 2008.

[25] J. Henderson, "Double solutions of impulsive dynamic boundary value problems on a time scale," Journal of Difference Equations and Applications, vol. 8, no. 4, pp. 345-356, 2002.

[26] J. Li and J. Shen, "Existence results for second-order impulsive boundary value problems on time scales," Nonlinear Analysis: Theory, Methods \& Applications, vol. 70, no. 4, pp. 1648-1655, 2009.

[27] D.-B. Wang, "Positive solutions for nonlinear first-order periodic boundary value problems of impulsive dynamic equations on time scales," Computers $\mathcal{E}$ Mathematics with Applications, vol. 56, no. 6, pp. 1496-1504, 2008.

[28] J.-P. Sun and W.-T. Li, "Positive solutions to nonlinear first-order PBVPs with parameter on time scales," Nonlinear Analysis: Theory, Methods E Applications, vol. 70, no. 3, pp. 1133-1145, 2009.

[29] R. W. Leggett and L. R. Williams, "Multiple positive fixed points of nonlinear operators on ordered Banach spaces," Indiana University Mathematics Journal, vol. 28, no. 4, pp. 673-688, 1979.

[30] J.-P. Sun and W.-T. Li, "Existence and multiplicity of positive solutions to nonlinear first-order PBVPs on time scales," Computers E Mathematics with Applications, vol. 54, no. 6, pp. 861-871, 2007. 